\title{
A combined three-dimensional digitisation and subsurface defect detection data using active infrared thermography
}

\author{
by M. Belkacemi* , C. Stolz*, A. Mathieu**, G.Lemaitre* and O. Aubreton*
}

*Université Bourgogne Franche-Comté, LE2I UMR6306, CNRS, Arts et Métiers, 12 rue de la Fonderie, Le Creusot 71200, France

** Université Bourgogne Franche-Comté, ICB, UMR 6303 CNRS, 12 rue de la Fonderie, Le Creusot 71200, France

\begin{abstract}
In recent years, NonDestructive Testing (NDT) systems have been upgraded with three-dimensional information. Indeed, combine the three-dimensional and thermal information allows a more meaningful analysis. In the literature, the data for NDT and three-dimensional (3D) reconstruction analysis are commonly acquired from independent systems. However, the use of two such systems leads to error analysis during the data registration. In an attempt to overcome such problems, we propose a single system based on active thermography approach using heat point-source stimulation to get the 3D digitization as well as subsurface defect detection. The experiments are conducted on steel and aluminum objects, and a combined 3D / thermal-information is presented.
\end{abstract}

\section{Introduction}

Active thermography is a noncontact and a non-invasive technique for NDT. This technique consists to carry out a thermal perturbation on the object surface in order to produce a thermal contrast between the defective and non defective area. Numerous methods have been proposed for different applications such as Lock-in Thermography [1, 2], Pulsed Thermography [3], Pulse Phase Thermography [4], Square Pulse Thermography [5]. These methods are robust when the surface inspected is planar. However, in the case of non planar object, the signal distortion leads to error analysis. A short time ago, an evolution of the NDT systems by adding 3D measurement has been noted. Fernandes et al. study the benefit of using multi-modal information to assess the fiber orientation [6]. They have shown that mapping InfRared (IR) information with the 3D model lead to a better estimation of the fiber orientation than IR information lonely. Similarly, Oswald-Tranta et al. have detected cracks in nonmagnetic materials based on the fusion between the IR and the 3D data [7].

NDT analysis combined with 3D information is usually performed by the fusion between the data collected from a 3D scanner and a non-destructive system. However, beyond the financial aspects, the use of two systems poses the problem of data registration. The alternative would be to use the same data for 3D reconstruction and NDT analysis.

In the literature, Scanning From Heating (SFH) system is presented as an active thermography technique which allows to get the 3D geometry of metallic object [8]. The method consists, firstly, to calibrate a galvanometer scan head and an IRcamera using the calibration proposed by Marzani et al. [9].

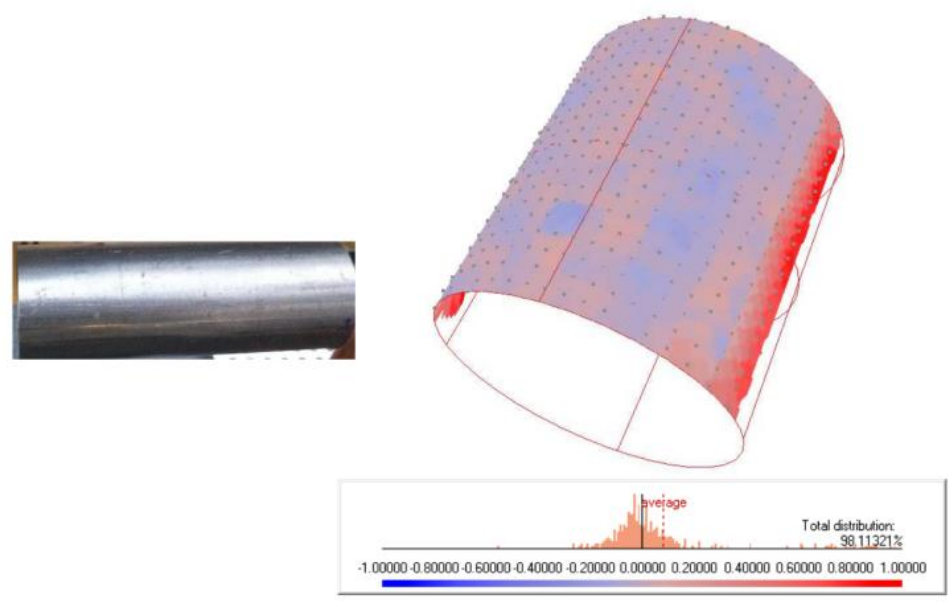

Fig. 1. 3D cylinder digitization [10]. 


\subsection{1/qirt.2016.006}

Secondly, a laser scan is performed on the object surface and for each scan an IR-image is acquired. Finally, the infrared radiation captured by the IR-camera is quantified by an in-house image processing algorithm and the 3D information is obtained through a triangulation. The mean error of the reconstructed surface is $70 \mu \mathrm{m}$ with a standard deviation of $71 \mu \mathrm{m}$ (see Fig. 1).

On the other hand, several studies using heat point-source stimulation for non-destructive testing can be found. Burrows et al. have detected cracks defects on an object surface [11], they used a punctual stimulation (a laser) to scan the surface object using a laser, and any change in thermal profile detected refers to the presence of crack.

Likewise, Ermert et al. have developed a noncontact detection approach based on a Scanning Electron Microscope (SEM) in order to detect the subsurface defects [12]; a modulated electron beam is used to stimulate the surface and the thermal response is captured by a pyroelectric device. Indeed, the amount of IR radiation increases and allows to localize the defect, when the scan is on a defective area

In this work, we present an approach based on SFH system in which heat point-source stimulation is used, the 3D geometry and NDT analysis are obtained through a specific image processing and with the same infrared data. Once the 3D model and NDT analysis are obtained, a data fusion is performed between these two modalities.

The proposed method is an active thermography technique and is explained in Sec. 2 while the experimental setup and results are presented in Sec. 3. Finally, the conclusion and future works are discussed in Sec. 4.

\section{Proposed method}

First of all, The SFH system consists of a galvanometer scan head and IR camera. The system is calibrated as proposed by Marzani et al. [7]. The method consists of translating a plane perpendicularly to the central axis of the laser beams projector. An image is acquired for each laser scan and this procedure is repeated for each successive translation. Then, the locations of these dots on the IR-image are extracted for every single acquisition. Subsequently, a relationship between each laser beam and its projection on the IR-image is calculated.

Once the system is calibrated, the surface object under study is point-to-point scanned using laser stimulation. To carry out this task, the mirrors of galvanometer scan-head are controlled by the SamLight software, by specifying the $\mathrm{x}$, y coordinates of the stimulation direction.

At each of these scans, a thermal image is acquired before and after the stimulation. The collected IR data are used to achieve two task: (i) 3D reconstruction and (ii) subsurface defect localization.

For the first purpose, the method is well described in $[13,14]$. The only difference lies in the detection of the thermal response center. Indeed, the Pre-processing and Segmentation steps described thereafter are considered to segment the IR-image. The ellipse edge on segmented IR-image is determined using the Sobel filter [15].

Finally, the centroid of the segmented ellipse is found by fitting an ellipse through a least square minimization [16].

Regarding the defect detection, the heat distribution is disturbed by the presence of subsurface defect. An image processing dedicated to identify changes of the heat distribution is developed. The framework is summarized in Fig. 2 and consists of:

(i) Pre-processing in which the acquired thermal image is enhanced by subtracting the background and reducing the noise using a median filter.

Segmentation in which the enhanced image is segmented using the region growing segmentation algorithm [17]. Indeed, the seed is placed where the temperature is maximum; the region grows if the difference of the neighboring intensity pixels and the calculated mean region is less than a specific criterion determined empirically.

(iii) Detection in which all segmented images are combined together by creating a matrix "M" such that the number of white pixels of a given segmented image is assigned to the location $M(i, j)$, where $i$ and $\mathrm{j}$ correspond to the location of the laser beam.

At this stage, the 3D geometry of the surface and the subsurface defect detection are determined. Since the data used to achieve these two tasks are acquired from the same system, the results are expressed in the same coordinate system this fact allows us to merge the two results in a simple way by mapping the $2 \mathrm{D}$ defect detection texture on the 3D model of the object.

\section{Experimental setup}

The experimental setup is depicted in Fig. 3; the system is composed of:

1) A FLIR $645 \mathrm{IR}$ camera with a sensitivity range from 7.5 to $14 \mu \mathrm{m}$, the IR camera acquires a $640 \times 480$ pixels image. 


\subsection{1/qirt.2016.006}

2) An Nd: YAG laser of $1.06 \mu \mathrm{m}$ wavelengths is coupled with a galvanometer scan-head of two mirrors to control the position of the laser beam, the angle between the IR Camera and the galvanometer scan head is approximately equal to $45^{\circ}$. The collimated laser beam passes through the two mirrors of the scan head and projected on the inspected surface.

The surface to inspect is placed at a distance of $50 \mathrm{~cm}$ from the output focal. Indeed, the optical system is equipped with a lens focal length of $500 \mathrm{~mm}$ which allows obtaining a beam waist of $0.44 \mathrm{~mm}$ at the previously mentioned distance.

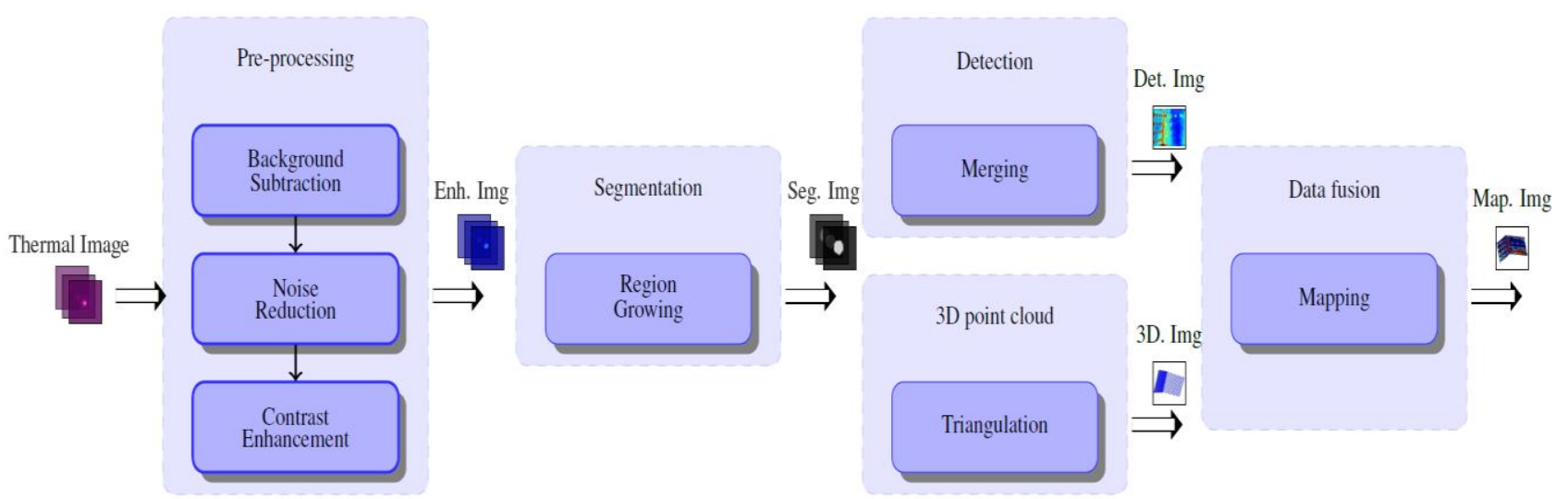

Fig. 2. Algorithm work flow.

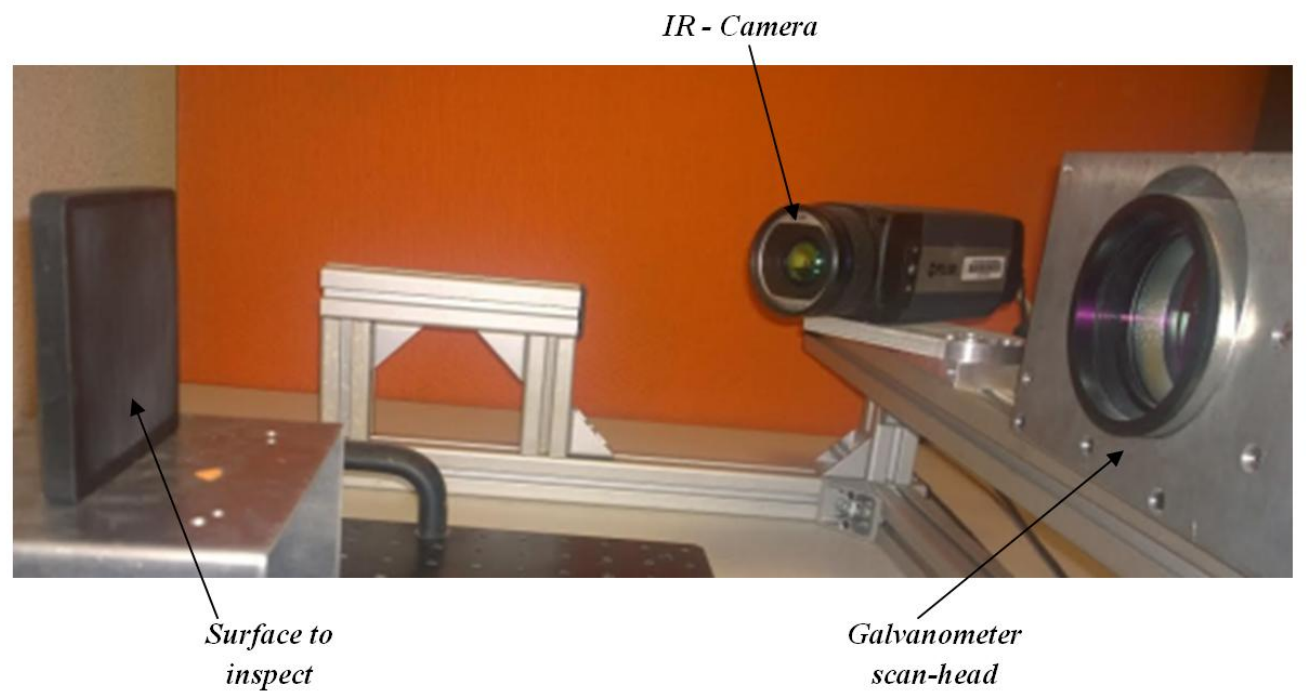

(a)

Fig. 3. (a) Experimental Setup.

\section{Experimental results}

We first analyze the defect detection algorithm. The experiments have been conducted on steel (see Fig. 4(a)) and aluminum plate (see fig. 4(b)). The steel plate contains circular subsurface defect of $0.5 \mathrm{~mm}$ diameter at different depths (between 0.5 and $2 \mathrm{~mm}$ ). The aluminium plate contains subsurface defect of different shapes and at different depths ( 0.5 to $2 \mathrm{~mm}$ ). The laser power is set up at $2.5 \mathrm{~W}$.

The IR data are processed with the algorithm described in Sec. 2. The inspected plates and their correspondent detection-maps are depicted in Fig. 4. Each pixel value of the detection-map represents the numbers of white pixel on the segmented image (Detection step of the algorithm). The pixels values of the detection-map are normalised between 0 and 1 and displayed with a jet color-map, where the bleu corresponds to 0 and the red to 0.8 . 


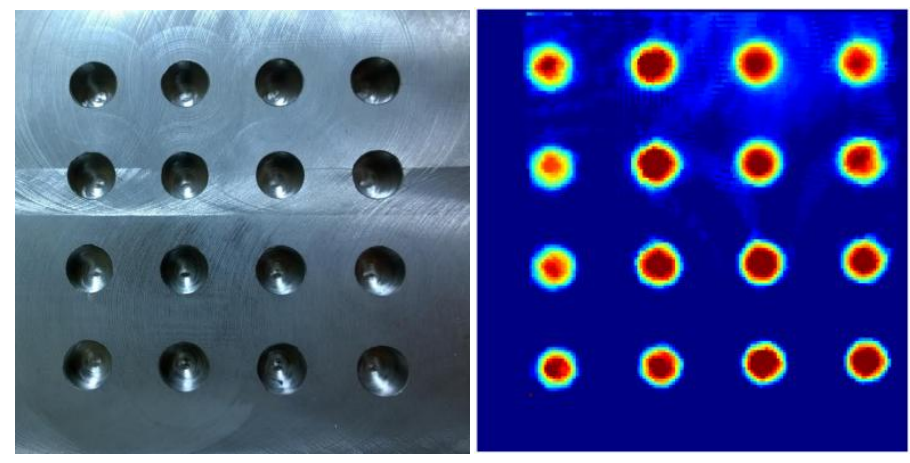

(a)

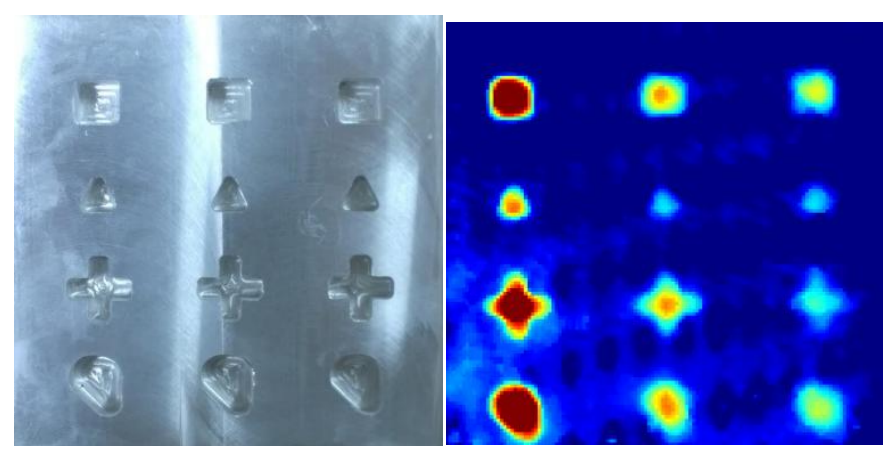

(b)

Fig. 4. (a) Defect detection on steel plate, (b) defect detection on aluminium plate.

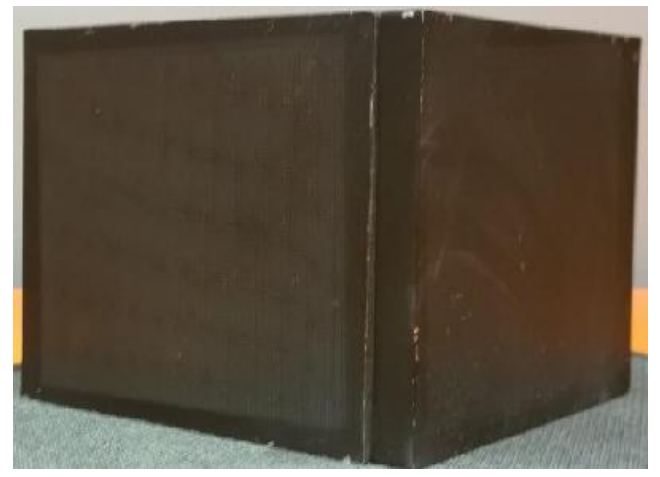

(a)

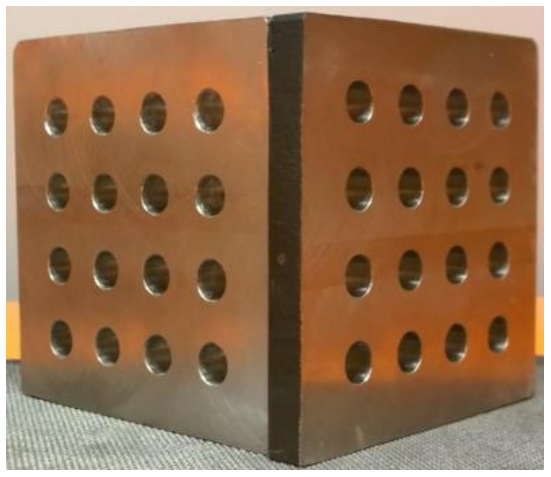

(b)

Fig. 5. (a) The front side of the cubic object made of steel, (b) the backside of the cubic object made of steel.

For the steel plate, we can easily distinguish the location of the subsurface defect from the non-defective area, and all defects at different depth are detected.

In another hand, the experiment conducted on aluminium plate shows that all defect are detected. Although the image contrast is sufficient to distinguish the location of the subsurface defect, the contrast decreases when the subsurface defects are deeper. Moreover, the shallowest defects have a greater intensity pixel (in red) than the deeper defects (in yellow).

The second step in our experiment is to test our algorithm on steel and aluminum object with a cubic form. The IR data are used to achieve successively 3D digitization, defect detection and data fusion.

For the steel object (see Fig. 4), the subsurface defects are circular of 9-mm diameter and located at different depths (between 0.5 and $2.5 \mathrm{~mm}$ ). 


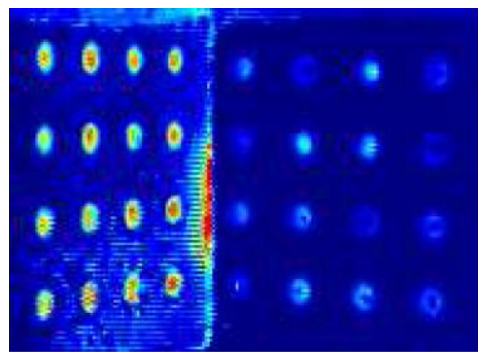

(a)

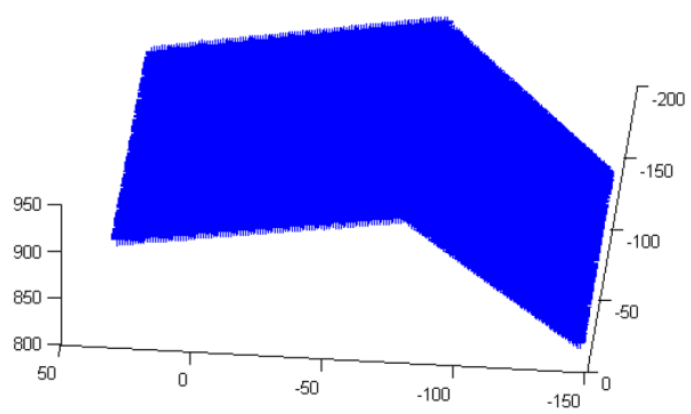

(b)

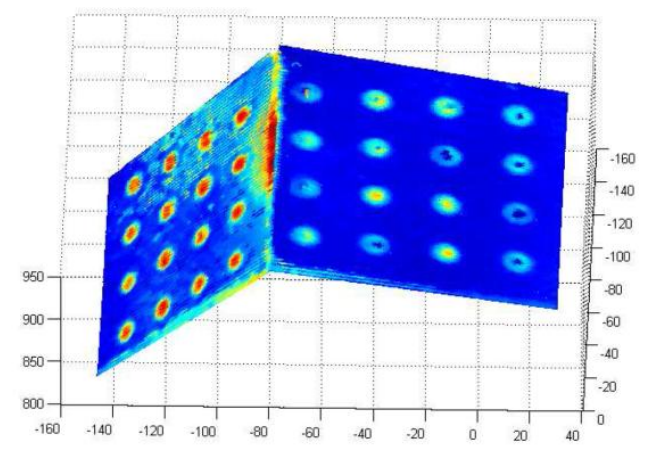

(c)

Fig. 6. (a) Detection map (NDT-texture), (b) 3D model, (c) 3D / NDT data fusion.

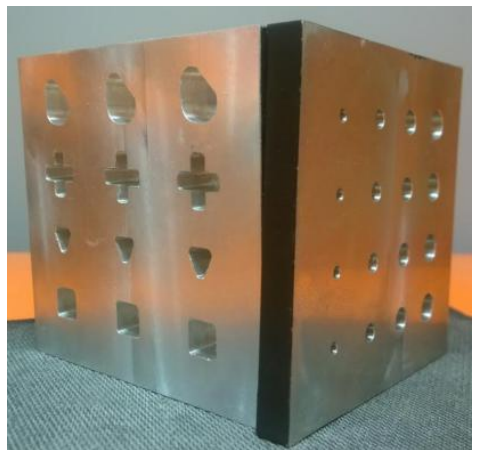

(a)

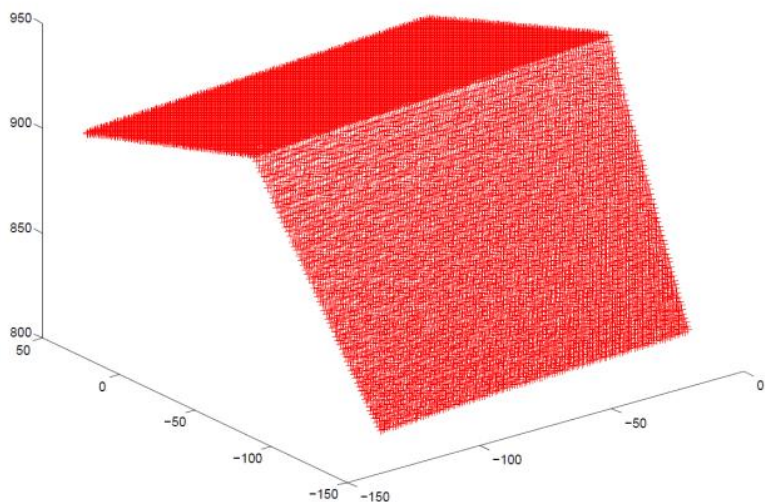

(c)

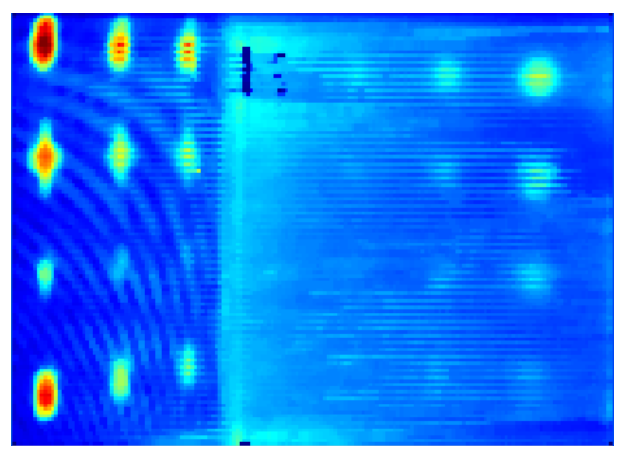

(b)

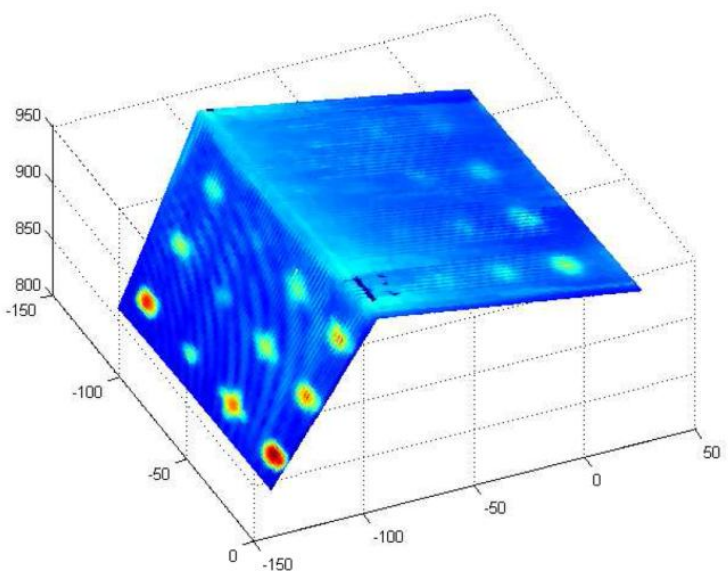

(d)

Fig. 7. (a) The backside of the cubic object made of aluminium, (b) corresponding detection map (NDT-texture), (c) 3D Model, (d) 3D / NDT data fusion. 


\subsection{1/qirt.2016.006}

For the aluminium object, the subsurface defects have different shapes and are located at depths between $0.5 \mathrm{~mm}$ and $3 \mathrm{~mm}$. Moreover, the circular subsurface defects have different diameter (form 3 to $9-\mathrm{mm}$ ).

Figure 6 and 7 show the results obtained on the steel and aluminium objects respectively. In the case of steel object, all circular subsurface defects are detected. The data fusion between the 3D data and NDT data is depicted in Fig. 6 (d).

Figure 7 shows the results obtained on aluminium object, all subsurface defect less than $2.5 \mathrm{~mm}$ depth are detected. Circular defect with diameter less than 6-mm are not detected. We can also observe the subsurface defect shape. The data fusion between the 3D data and NDT data is depicted in Fig 7(d).

\section{Conclusion}

In this paper, an approach based on heat point-source stimulation was proposed to detect subsurface defects and get the 3D geometry of the surface using same infrared-data acquired. To achieve this goal, an algorithm which exploits changes in the thermal response between defective and non-defective area was proposed. To validate our method, experiments were conducted on steel and aluminium plates. The results highlighted that subsurface defects up to $2.5 \mathrm{~mm}$ depth are detected and the shape of the defect can be observed.

In another hand, the use of the same IR data which is expressed in the same system coordinate avoids problems related to data registration. This allows achieving a data fusion between the $3 \mathrm{D}$ digitization and the subsurface defects detection data by mapping the 2D-NDT texture on the 3D model of the surface, directly.

As future work, we will investigate the possibility to enhance the signal-to-noise ratio. Another avenue of research lies in the study of the detection of surface defects such as cracks. And size, depth quantification by using the temporal evolution of the thermal response.

\section{ACKNOWLEDGMENTS}

This work is supported by the Regional Council of Burgundy. We are extremely grateful to members of laboratory LE2I for day to day help and collaboration.

\section{REFERENCES}

[1] Busse, G., Wu, D. and Karpen, W. , "Thermal wave imaging with phase sensitive modulated thermography," J.Appl.Phys. 71(8), 3962-3965 (1992).

[2] Wu, D. and Busse, G. , "Lock-in thermography for nondestructive evaluation of materials," Revue générale de thermique 37(8), 693-703 (1998).

[3] Ibarra-Castanedo, C., Genest, M., Servais, P., Maldague, X. and Bendada, A. , "Qualitative and quantitative assessment of aerospace structures by pulsed thermography," Nondestr.Test.Eval. 22(2-3), 199-215 (2007).

[4] Maldague, X. and Marinetti, S. , "Pulse phase infrared thermography," J.Appl.Phys. 79(5), 2694-2698 (1996).

[5] Vavilov, V., Kauppinen, T. and Grinzato, E. , "Thermal characterization of defects in building envelopes using long square pulse and slow thermal wave techniques," Journal of Research in Nondestructive Evaluation 9(4), 181-200 (1997).

[6] Fernandes, H. and Maldague, X. , "Fiber orientation assessment in complex shaped parts reinforced with carbon fiber using infrared thermography," Quantitative InfraRed Thermography Journal 12(1), 64-79 (2015).

[7] Oswald-Tranta, B. and O'Leary, P. , "Fusion of geometric and thermographic data for automated defect detection," Journal of Electronic Imaging 21(2), 021108-1-021108-8 (2012).

[8] Aubreton, O., Bajard, A., Verney, B. and Truchetet, F. , "Infrared system for 3D scanning of metallic surfaces," Mach Vision Appl 24(7), 1513-1524 (2013).

[9] Marzani, F. S., Voisin, Y., Voon, Lew FC Lew Yan and Diou, A. , "Calibration of a three-dimensional reconstruction system using a structured light source," Optical Engineering 41(2), 484-492 (2002).

[10] Bajard, A. Numérisation 3D de surfaces métalliques spéculaires par imagerie infrarouge (2012).

[11] Burrows, S. E., Dixon, S., Pickering, S. G., Li, T. and Almond, D. P. , "Thermographic detection of surface breaking defects using a scanning laser source," NDT E Int. 44(7), 589-596 (2011).

[12] Ermert, H., Dacol, F., Melcher, R. and Baumann, T. , "Noncontact thermal-wave imaging of subsurface structure with infrared detection," Appl.Phys.Lett. 44(12), 1136-1138 (1984).

[13] Bajard, A., Aubreton, O., Fofi, D., Morel, O., Truchetet, F. and Meriaudeau, F. , "Non conventional Imaging Systems for 3D Digitization of transparent and/or specular manufactured objects," QCAV2013, 11th Interntional Conference on Quality Control by Artificial Vision, 190-197 (2013).

[14] Bajard, A., Aubreton, O., Bokhabrine, Y., Verney, B., Eren, G., Ercil, A. and Truchetet, F. , "3D Scanning of specular and diffuse metallic surfaces using an infrared technique," Optical Engineering 51(06), 0091-3286 (2012).

[15] Matthews, J. An introduction to edge detection: The sobel edge detector (2002).

[16] Fitzgibbon, A., Pilu, M. and Fisher, R. B. , "Direct least square fitting of ellipses," Pattern Analysis and Machine Intelligence, IEEE Transactions on 21(5), 476-480 (1999).

[17] Adams, R. and Bischof, L. , "Seeded region growing," Pattern Analysis and Machine Intelligence, IEEE Transactions on 16(6), 641-647 (1994). 